\title{
COVID-19 Outbreak: Model-Driven Impact Analysis Comparing Oman and Pakistan
}

\author{
Kashif Zia ${ }^{1, *}$, Umar Farooq ${ }^{2}$, and Muhammad Shafi ${ }^{3}$ \\ ${ }^{1,3}$ Faculty of Computing and Information Technology, Sohar University, Sohar, Oman \\ ${ }^{2}$ University of Science and Technology, Bannu, Pakistan \\ * Corresponding author: Kashif Zia ${ }^{1}$, kzia@su.edu.om.
}

\begin{abstract}
Motivated by the rapid spread of COVID-19 all across the globe, we have performed simulations of a system dynamic epidemic spread model in different possible situations. The simulation, not only captures the model dynamic of the spread of the virus, but also, takes care of population and mobility data. The model is calibrated based on epidemic data and events as they happened. The simulation results are quite disturbing, indicating that, during a process of stringent social distancing and testing strategies, a small perturbation can lead to quite undesirable outcomes. The simulation results, although consistent in expected outcomes across changing parameters' values, also indicate a substantial mismatch with real numbers. An analysis of what can be the reason of this mismatch is also performed. Within these contradictions, a comparative analysis of COVID-19 outbreak between two geographically close but demographically very different countries - that is Oman and Pakistan - is performed.
\end{abstract}

Keywords: Coronavirus; COVID-19; epidemic model; simulation; impact analysis. 


\section{Introduction}

COVID-19 is the latest evidence of epidemic disease capable of producing an extraordinarily large number of infections starting from a few [1]. According to Lippi and Plebani, Coronavirus disease 2019 (COVID-19), which originated in the city of Wuhan China on December 01, 2019, is a respirational and zoonotic disease, caused by a virus of the coronaviridae family [2]. The Virus strain is severe acute respiratory syndrome coronavirus 2 (SARS-CoV-2), resulting in fever, coughing, breathing difficulties, fatigue, and myalgia. It may transform into pneumonia of high intensity.

Towards successful diagnostic and cure of COVID-19, scientists in the field of molecular biology [3] are working hard to find answers about its spreading and infecting by examining virus samples. Although, the disease strain is known, but the vaccine is nowhere near. And it is essential to ensure strict mitigation actions so that virus can be contained. Already, most of the countries are taking 15 different kinds of precautionary measures to cope with it so that the losses can be reduced. The worldwide outbreak of COVID-19 due to the SARS-CoV-2 (previously called 2019-nCoV) has put great pressure on governments across the globe to control the spread of infection. To begin with controlling this novel coronavirus, the world needs to get prepared for, and that's possible only if risk assessment is done based on sharing the transparent information. The assessment of the reports on outbreak site as well as those about investigation must be considered. Since, no treatment is available, the risk of spreading the infection could be mitigated by the experiences of the countries, who has reduced the risk [4].

In 2013, Severe Acute Respiratory Syndrome (SARS) claimed about 800 deaths among 8000 total cases reported, and this was contained finally due to prompt and strict actions including surveillance, isolation of patients, and enforcing quarantine of not only all contacts of the patients but also communities in some areas. Disrupting human to human transmission, SARS was successfully controlled within 8 months. SARS was restricted mostly to only 6 countries though it was reported in 26 countries. According to Wilder-Smith et al. [5], COVID-19 resulted in more than 82,000 cases and 2800 confirmed death in the initial two months. They pointed out that COVID-19 and SARS have striking similarities. They are not only same $86 \%$ genetically but have a high degree of homology. They are also similar in terms of disease dynamism. The transmission route for both is respiratory droplets. Both of them lead to severe infection in elderly people. However, the incubation period, the ability of transmission, spreading, and clinical severity in COVID-19 are different than SARS. According to the them, it will the differences not the similarities among the features of viruses, which measures will be successful to cope with COVID-19. The authors, however, 
believe that even if the traditional health measures used to cope with SARS are not used to fully eliminate COVID19, these measures will be effective in reducing the global cases and deaths. The countries must take rapid countermeasures to cope with this disease, and thus reducing large scale outbreaks, when the disease is exported to the rest of the world. They recommended that the measures which were taken to cope with SARS must be utilized, especially when very little is known about the novel coronavirus and in absence of vaccines to tackle it.

According to Sohrabi et al. [6], the disease which spread in Wuhan from sea food market (dealing in fish and live animals such as poultry, bats and snakes) in December 2019, was named COVID-19 by World Health Organization (WHO). The majority of infected patients had mild symptoms such as dry cough, fever, and sore throat. Many people have recovered, however, some of them had fatal complications such as failure of organs, septic shocks, severe pneumonia, and acute respiratory distress syndrome. COVID-19 was declared as a public health emergency by WHO on January 30, 2020, of international concern. This was due to the reason that this disease is posing a high risk to countries with weak heath systems. "WHO" stated COVID-19 spreading could be interrupted using by an early detection of the patients and their isolation, quick treatment, and robustly tracing the contacts of the infected people.

From the experiences of China and South Korea, the countries able to contain the spread of the disease so far, it is learnt that social distancing and testing are the key factors. Another factor responsible of spreading of the disease worldwide was regional and global travellers. Although, air travel is almost suspended now, but, this initial shock and countries (and people) not taking it too seriously has taken many countries in Europe and North America to a real bad situation.

According to Hellewell et al. [7], isolation of confirmed cases and tracing their contacts has been used in controlling infectious diseases and it is also used in current outbreak of COVID-19. To achieve the control using this strategy, however, depends on the characteristics of both the virus and response. They assessed the isolation and contact tracing technique for controlling the transmission of disease from the COVID-19 cases imported in the UK from China. They tested their model for various scenarios and concluded that in most of the scenarios, a highly effective tracing and isolation technique was enough for controlling the outbreak in 3 months. They learnt that long delays in isolation in those cases where the symptoms were detected late, an increase in disease transmission was observed.

In this overall scenario, after widespread suspension of air travels, many countries have taken various other measures to avoid spreading the infection. 
Schools and Colleges/Universities were closed, followed by other non-essential offices and services. The situation is been monitored on daily basis by all the governments. However, all across the world, there are many suspected cases which are not been tested so far. By the time of this writing, most of countries are in the state of partial or full lock down. In many countries, new cases are appearing continuously. And the next few week are very important. To know what may happen, we need to model and simulate. Towards this, we model the dynamics of spread of the disease (the epidemic model), population data and mobility of people. We have used a system that is designed to it.

The epidemic model presented in this paper is not novel. In fact, similar (or even same) models are already proposed in different fields of study. However, contextualization and implementation of the model in the current global and regional situation is significantly important. Already such studies are been taken up by the research groups working in this area [8]. Through this paper, we have provided a focused analysis of the situation and asked important what-if questions, particularly in the context of Oman and Pakistan. However, the suggested method can be applied to any other country of the World, or even at the global level. Also, we have tried to argue why there is a big mismatch between real cases and cases predicted by the simulation.

Countries like Pakistan have four problems which make them more vulnerable than others; (i) a huge congested population, (ii) lack of medical facilities, (iii) poverty, and (iv) culture. Although, the spread of the disease is not that much as of today (March 31, 2020), the new cases are appearing continuously and the next few week are very crucial. Pakistan had a population of 22 crore people mostly residing in densely populated cities and towns. In addition to congregational prayers (which are not yet banned), it has huge number of mosques where congregation prayers (5 times a day) are offered which are joined by huge population. There are very limited number of hospitals. Ventilators throughout the country are not more than 2000 , where $1 / 3 \mathrm{rd}$ of them are in private hospital. The health teams have shortage of safety and testing kits. Such problems mostly do not exist in Oman, at least not at that scale. Hence, a comparison between the two countries would give us a comparison between a complicated country versus a country which is not that complicated.

Contribution: We have proposed a system dynamic model of infectious disease spread and parameterized it factually according to real events for specific countries. The Global Epidemic and Mobility Model (GLEaM) was used to integrate the model so that different simulation scenarios can be tested. A detailed description of parameters and their usage is given so that a situation, a sequence of events, and an outlook of the future could be implemented. 


\section{COVID-19 Disease Spread Model}

\subsection{The Base Model}

In The model is based on well-established state-transition systems that are being used to study epidemics for a long time. The simplest one are SI (susceptible infectious) and SIR (susceptible-infectious-recovered) models [9]. In both, an infectious individual infects a susceptible individual at a rate $\beta$. In SIR model, we also have a recovery rate $(\mu)$ after which an infectious individual is recovered permanently.

It can easily be seen that simple SIR model does not fully grasp COVID-19. For epidemics like corona-virus, SIR model was extended to SEIR [10], introducing a new state exposed between susceptible and infectious. This state is also known as latent, representing the period during which the individual has been infected but is not yet infectious himself. Therefore, we also have an exposed rate denoted by $\varepsilon$.

Still, the model needs further extension. The closet model representing the COVID-19 specifications is the one proposed for H1N1 epidemic [11]. Like in H1N1, in COVID-19, we have two types of infectious individuals; one that show symptoms (Symp) and the other who do not show symptoms (ASymp). And, an exposed individual can transit to state infectious Symp with rate or to a state infectious ASymp with rate 1- $\varepsilon$.

\subsection{The COVID-19 Model}

The base model adapted from [11] is further extended to incorporate a new state isolated or quarantine. The isolated state represents the possibility of transferring an infectious individual with symptoms to isolation with a rate $\alpha$. The value of $\alpha$ is then used to represent preparedness of health system of a country (or globe). All three states infectious Symp, infectious ASymp, and isolated, transits to recovered state with same rate $\mu$. Also, a transition from infectious ASymp to infectious Symp is made possible with a rate $\rho$. The final model is shown in Figure. 1.

\section{Model Implementation}

\subsection{GLEAMviz Simulator}

The model is implemented in GLEAMviz [12], the global epidemic and mobility model. GLEAMviz is a simulator which uses real-world data of population and mobility networks (both airways and commuting) on the server side. It integrates this data with the model developed by a user on the client side (similar to what we have presented in Figure. 1). 


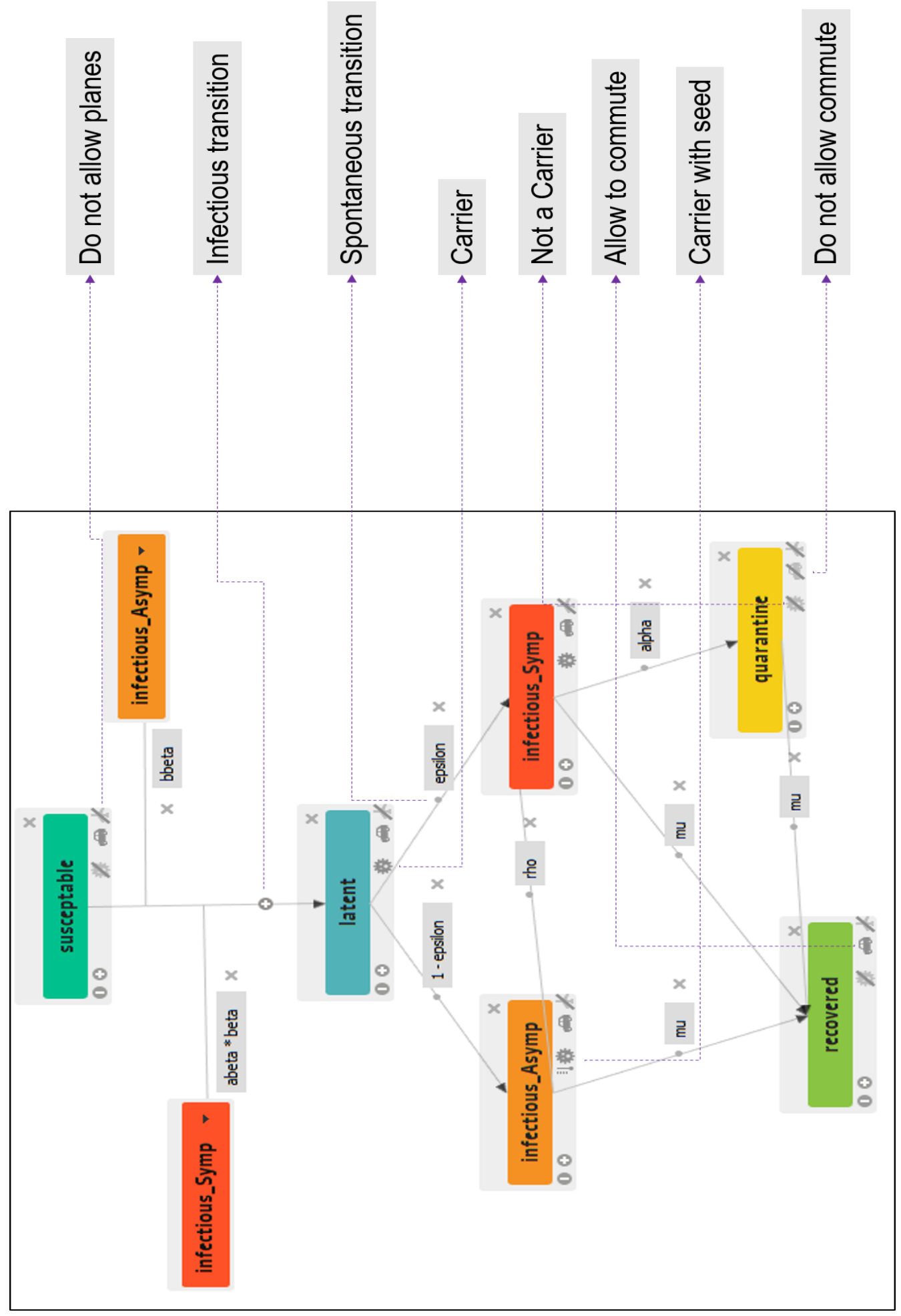

Figure1: The COVID-19systemdynamicsmodel. 
Hence, the simulation generated in data driven, in which a user is responsible of describing system dynamics model of the epidemic, whereas, all the relevant population and mobility global data in integrated at the server side. As a result, the time-series data of spread of epidemics is generated by the server system.

In GLEAMviz client, the model is developed by showing transitions between different compartments (states). The model, conceptualized in the previous section, built on compartments and transitions is shown in Figure 1. One aspect not explained yet is that there are two type of transitions. The infectious transition is represented by "+" sign, depicting an addition of infectious cases. There is only one instance - from susceptible to exposed/latent - like that. The other transitions are spontaneous (represented by dot sign). The initial seed to the model is provided at compartment infectious Asymp, that is, few initial effects as starting seed that do not possess any symptoms.

\subsection{Model Specifications}

They are two functional modes of the model.

\subsubsection{Carrier-ship and Mobility}

An individual who is exposed to the virus (whether with symptoms or without) is the carrier of the virus. Therefore, a susceptible and recovered individual is not a carrier, while others are. Although, technically, an isolated/quarantined individual is a carrier, but we have assumed that she/he is quarantined and is no longer able to transmit. Next are mobility possibilities. We have mimicked very recent situations to restrict or allow mobility. For example, considering all air traffic suspended, no compartment allows air travel. Whereas, all individuals who are not quarantined are allowed to commute locally. The commuting 185 restrictions are further modified by using different transition variables.

\subsubsection{Transition Rates}

The following are the transition rates from one compartment to another. Note, that there are quite a few refinements in the base model. Also, for a Greek alphabet, it's symbol and text are used interchangeably. All rates vary between 0 and 1 , inclusive. 
- beta: infectious rate that transforms susceptible to exposed. This happens under the influence of both individuals with or without symptoms. To differentiate, we have taken bbeta as the $\beta$ value for individual with no symptoms, and abeta $\times \beta$ as the value for individual with symptoms.

In this way, we are able to relate the infections incurred in different situations.

- epsilon: rate of transiting from exposed to infectious state. An exposed individual can transit to state infectious Symp with rate or to a state infectious ASymp with rate 1-E. The value of is reciprocal of exposed period, which is equal to 5.2 days in our model [13].

- rho: rate of transiting from infectious ASymp to infectious Symp state. The value of $\rho$ is reciprocal of symptoms appearing period, which is equal to 2.3 days in our model [13].

Simulation Setup and Results rate of transiting from being infectious or isolated to recovered. The value of $\mu$ is reciprocal of infectious period. It is taken 30 days in case of COVID-19.

- alpha: rate of transiting from infectious Symp to isolated state.

\section{Simulation Setup and Results}

The variations in beta, abeta, bbeta, and alpha make up different cases corresponding to different situations. The other variables (epsilon, rho and $\mathrm{mu}$ ) are kept constant. Variations are introduced systematically, based on what is observed in the last one month and what is being done by the governments. Based on the simulation results that we got, we categorized different situations (cases), which are: extremely bad (case 1), extremely good (case 2), and intermediate (case 3).

\subsection{Complete Inaction: Case 1}

Response What can be worse than a complete inaction by the authorities? The default values assigned to the variables: beta $=$ bbeta $=0.5$, abeta $=1$, and alpha $=0.001$, were able to generate such a situation. In this case, abeta $\times$ beta $=1.0 \times 0.5$ (infectious rate incurred by infected individuals with symptoms) and bbeta $=$ 0.5 (infectious rate incurred by infected individuals with no symptoms) both are 0.5 , depicting the basic setting with no differentiation. The fact that the rate of getting isolated in really low (alpha $=0.001$ ), depicts that there is no effort yet put by the authorities to contain the epidemic. In the context of Oman, we have put a few cases in Muscat, 
Khasab, and Salalah as the starting cases which got symptoms. Similarly, for Pakistan, we have put a few cases in Islamabad, Karachi and Gilgit as the starting cases. The simulation was run for time period of one year, starting from February, 26, 2020 (when a few such cases were reported in the both countries). Even though the infection rate $(\beta)$ is intermediate (only one out of two susceptibles are infected) and all flights are suspended (there are no outside influence), the results about spread of epidemic turned out to be really bad.

The results of case 1 are shown in Figure 2.

\subsubsection{Results: Oman}

The for Oman, the results suggest that the outbreak would be rapid and extreme, reaching 0.2 million cases (nearly $4 \%$ of the population) per day after 45 days of the outbreak, and then it would start dropping rapidly. When accumulated, it was noted that $80 \%$ of the population would be affected.

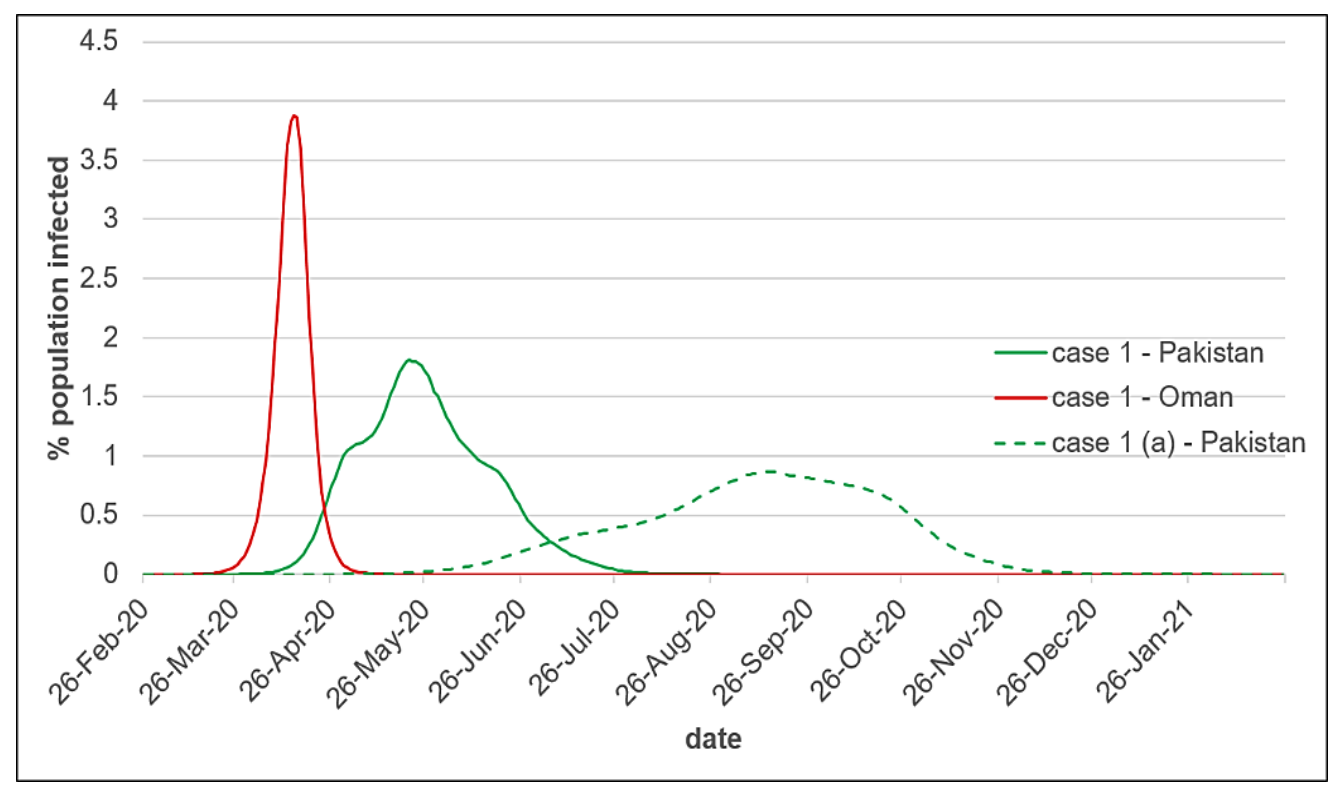

Figure 2: Infections (with symptoms): case 1

\subsubsection{Results: Pakistan}

For Pakistan, the results suggest that the outbreak would be rapid and extreme, reaching 4 million cases (nearly $2 \%$ of the population) per day after 75 days of the outbreak, and then it will start dropping. When accumulated, it was noted that $80 \%$ of the population would be affected. 


\subsubsection{Comparison}

A comparison between the curves shows that the intensity of outbreak is more intense and constraint within a short period in case of Oman, when compared to Pakistan. Why Oman is so different from Pakistan? One reason can be the lack of data in the simulator's database itself. Simulator only has three cities of Oman. We did not notice much mobility across the populated coastal area. That can be one reason that the epidemic did prolong for only 60 days as opposed to Pakistan which extended to 5 months. Still, the number of people infected in both cases is same. Does $80 \%$ population of Oman reside in only three cities? This clearly indicates problems in data used by the simulator (particularly in case of Oman). However, this discrepancy works in favor of Oman, and we can think of it as an optimistic outcome, for this case and for all other cases given below.

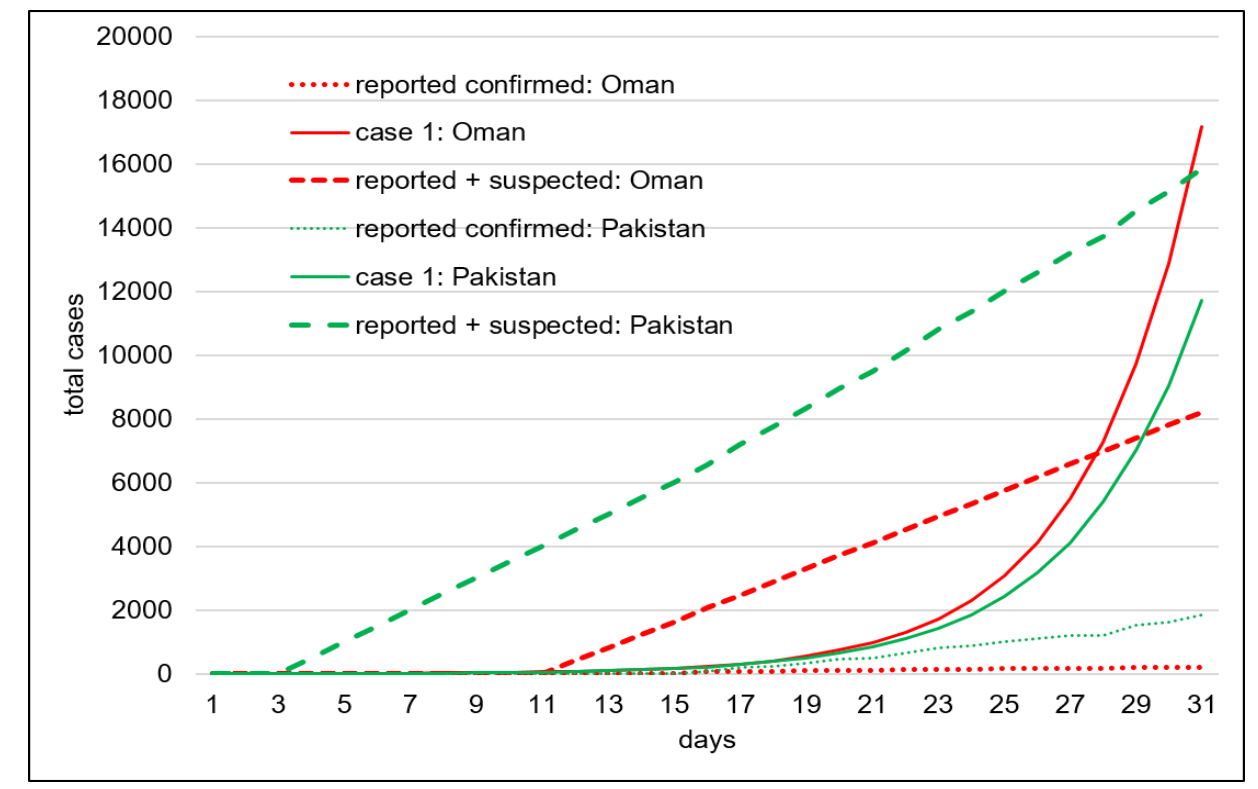

Figure 3: Infections (with symptoms) - with suspected cases: comparison first 30 days

\subsubsection{Real vs. Simulator's numbers}

A comparison between real cases reported for the first 30 days and what the model generated (in case 1) is given in Figure. 3. There is a clear mismatch. One reason can be the lack of testing. As of today (March 31, 2020), Oman as well as Pakistan still have several thousand suspected cases (the cases without testing). According to the [14], on May 
31, 2020, Oman had around 8000 suspected cases and that of Pakistan had 14000. If we just distribute this number equally after the first few days, and add them with the reported cases, we get a new curve (shown in Figure 3). This curve is very close to simulated results. In case of Oman, it both lines cross each other at day 28 . Hence, the simulation results generated correspond to the reality (at least for first 30 days). Nevertheless, we are not going to consider this inaction as reality after the actions been/being taken by the governments.

\subsection{Extremely strict actions: Case 2}

In this case, we try to mimic a forced isolation and a limited lock-down (enforced social distancing). This happens one week after the identification of the first cases. Many countries tried to put such restrictions for two weeks only, which were extended later on. We reproduced that by imposing if from March 3, 2020, but for 45 days. GLEAMviz provides an option to put exceptions in the form of rules to be applied for certain time period and for certain locations. The rules relate to setting the values based on mathematical expressions. For the above stated 45 days, we created exceptions, which are:

$$
\text { abeta }=0.05 \text {, bbeta }=0.05 \text {, and alpha }=0.95 \text {. }
$$

What these values mean? The alpha $=0.95$ means that infectious symp to isolated rate is $95 \%$ leaving only $5 \%$ patients to infect others. However, there is less probability of that happening due to lock down. Hence, we multiple abeta with beta to reduce it. bbeta is also reduced due to this reason. However, these reductions are quite strict and would be relaxed a bit in case 3. These exceptions were applied all across the country. The results of case 2 indicated that quite a few cases appeared in first week or so and then epidemic was eradicated. Case 1 and 2, both are unrealistic, however, they educate us about extreme situations.

\subsection{Realistic scenarios: Case 3}

The real problem with this virus is that it may infect others from patients who do not have any symptoms. The authorities always report confirmed case. They are usually quarantined. But the threat is always there from people who are infected and, in the society, undetected. To represent all this, we created the following exceptions:

- At the start of the simulation, we introduced a few patients with symptoms and same number of patients without symptoms, at the three cities mentioned for both countries. 
- The following exceptions were applied (based on real events): after the first three weeks, real isolation activity was realized, first in small cities and after further three weeks, all across the country, by setting alpha $=0.95$, and abeta $=0.05$.

- A complete lock down was implemented after one month by setting bbeta $=0.05$. But then the lock down was partially relaxed, after two weeks by setting the values of abeta and bbeta to 0.1 for rest of the simulation time.

Further, we introduced bulk cases (patients travelling and entering into the country). Initially many of these cases were gone undetected. These people were integrated with their families and all of them were infected without any symptoms. Hence, the results of this case (case 3) can be considered as nearest to reality according to current actions of the authorities. Unfortunately, the results of the simulation were not good as well, but not as bad as case 1 .

\subsubsection{Results: Oman}

As shown in Figure 4, we can see that the daily confirmed cases with symptoms reached to its peak of $1 \%$ of the population right at day 30 . Then they started to drop and reached to 0 at day 60 . The expected population that would be affected is really low when compared to case 1; a total of $16 \%$ of the population. Irrespective of the discrepancy between the reported cases and simulated ones, its good news for Oman. We are almost at the peak now and bracing for a downhill trajectory. Definitely, the containment policies should continue for 320 at least one more month, hoping that epidemic does not start its second cycle.

\subsubsection{Results: Pakistan}

The significance of the curve for Pakistan shown in Figure 4 is that the exponentiation of the cases starts very late, almost after six months and that it reaches more than 1 million cases (almost $0.5 \%$ of the population) per day. The expected population that would be affected is almost half of case 1 though; a total of $40 \%$ of the population. 


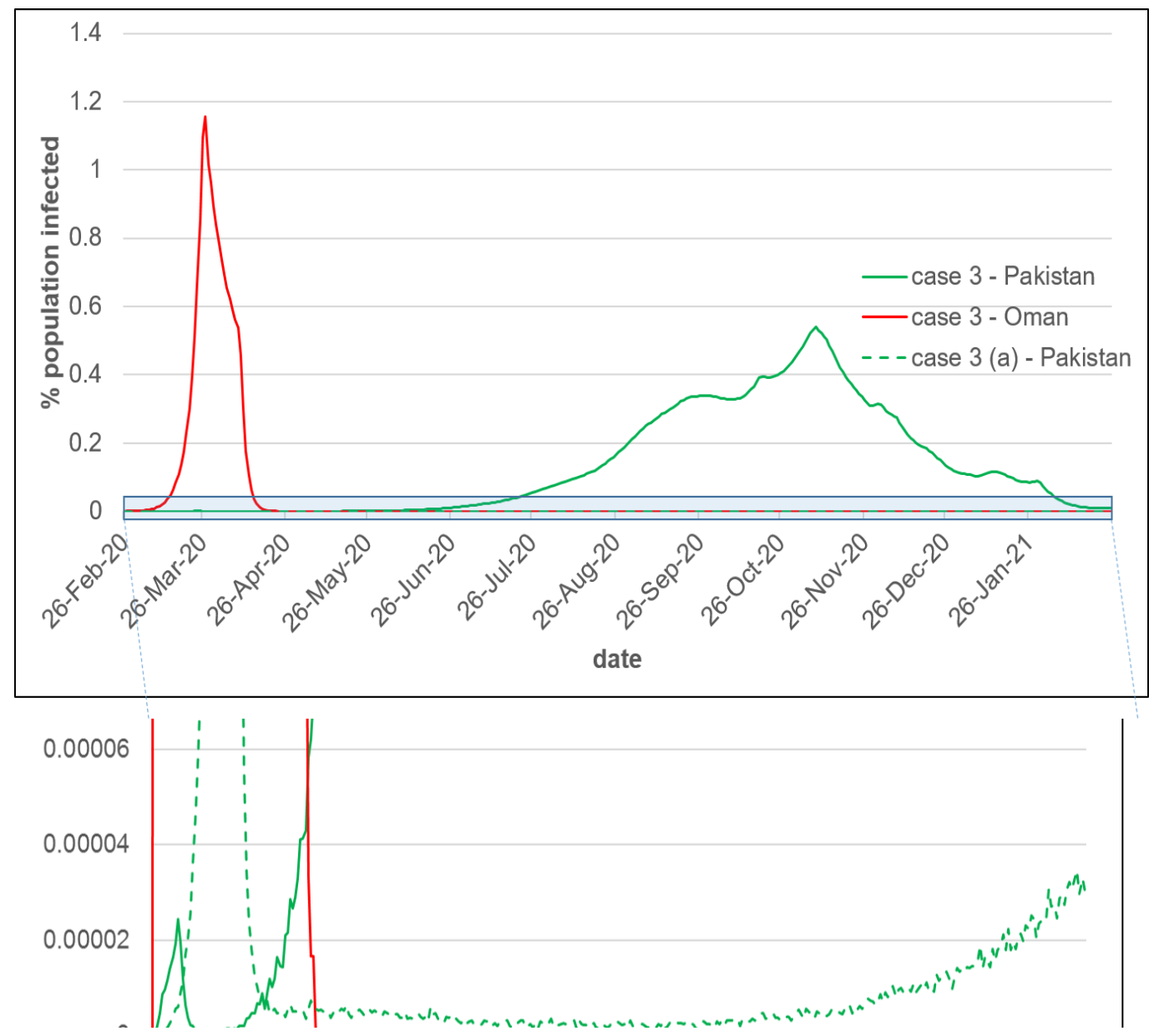

Figure 4: Infections (with symptoms): case 3

\subsubsection{Comparison}

Many countries are fearing about a large number of infections. Comparing case 1 with case 3 , we can deduce that whatever we do, the number - although much more manageable than the worst case - cannot be reduced dramatically. However, restrictions give us enough time (particularly for Pakistan). The model does not predict deaths. But, if we have time, before a real exponential growth happens, we can better prepare ourselves to reduce fatality rate.

\subsection{Optimistic scenario: weather interventions: case 1 (a)}

Although, we are not sure about it, but there is a high probability that the infections rate would drop when the weather warms up. Looking at bad situation in Pakistan, we created such situation using exceptions. This was achieved by reducing infection rates of the base case, case 1 . In the first case, beta and bbeta was set to 0.25 (half of the current 
infection rate) represented as case 1 (a). In the second case, beta and bbeta was set to 0.05 (almost none) represented as case $1(b)$.

Unfortunately, case 1 (a) did not have much impact. This meant that infection rate of 0.25 is still high (see Figure 2). But the trend was clearly visible. And, also, it has shifted the curve of case 1 (a) to the left when compared to case 1. Further reducing the infection rate would have more impact. But again, the authenticity of warm and humid weather disintegrating the virus remains to be proved. Nevertheless, if it is proved and the impact is significant, the epidemic would die on its own.

\subsection{Optimistic scenario: Longer lock down: case 3 (a)}

Looking at bad situation in Pakistan, we need to take actions at an extended time scale. Many countries in the World are now aware that a lock-down of a few weeks would not be sufficient. Therefore, in this case, we also extended the lock-down indefinitely after first month. Being optimistic, we hope a complete lock down in coming weeks (that is from end of March) for a longer period than 45 days all across the country. Thus, the value of bbeta would remain 0.05 from today on wards. But, if we will be able to provide sustained health services all across the country by identifying and isolating patients at villages level, the value of abeta can also be 0.05 for the whole year. We name this case as case 3(a). By looking at the graph of case 3 (a) in Figure 4, it is evident that at the start (almost as of today), the maximum has already reached at around 2200 cases a day (see inset 1 of Figure 4). After following the curve downwards, it almost stabilizes to 20 to 40 new cases per day later on (see inset 2 of Figure 4). This is quite manageable. But, it continues for a longer period, probably, beyond a year.

\section{Discussion}

Like most countries around the World, the authorities took actions against the spread of dangerous COVID-19 epidemic. Hence the first case (case 1) of unbounded spread, affecting almost $80 \%$ of population can easily be ignored. The epidemic is becoming more and more dangerous, partially due to absence of strict actions early on. The actions required are social distancing and healthcare management (including testing and isolation of patients). A really strict action towards this is not possible as evidenced around the world. Hence the case 2 was also ignored. 
In case 3, we implemented some real scenarios. We have seen that in case 3, in case of Oman, the epidemic's peak is near, followed by a decline towards its eradication. However, we should be careful about it due to two reasons. There is a substantial number of suspected cases, which have not neither been tested positive or negative. How many of these people are truly quarantined would be a deciding factor. The second aspect of it is inward traveling. As observed in China, a new wave of spread of epidemic may start due to people traveling into the country. Representing both these situations, as we can see in case 3, just a dozen of these cases may have a big impact. The simulator that was used has apparent limitations. There are only three cities for which the simulator has data. Also, during the running of the simulation, it was observed that mobility between the cities is almost non-existent. It is understandable, considering that cities of Muscat, Khasab, and Salalah are at the three corners of the country. With a more representative population and mobility data about different regions of the country, we may see more cases and a longer impact. In fact, it was surprising to see that Oman has an epidemic timeline of only two months, which cannot even be imagined in current unfolding of the events. We performed a simulation with similar kind of settings for Pakistan and found out that the impact may extend to 8 to 9 months [15].

To be more specific, in case of Pakistan, case 3 shows that $40 \%$ of population got infected, just due to inclusion of undetected bulk cases in the population. But the spread was delayed giving some time to prepare for it. Still peak of 1 million cases a day is a real nightmare. Consequently, we went for an indefinite lock down. Even, if the number of cases reached to 2000 a day (today on March 31,2020) - which can be true just due to overwhelmingly large number of suspected cases - we saw a continuous decline after today. However, the restriction could not be lifted. As seen in other cases, when the restrictions were lifted after 45 days, the rate of spread of disease become exponential again.

\section{Conclusion}

With a system dynamic model of epidemic spread, incorporated with population and mobility data, we performed simulation of many different cases of COVID-19 impact, representing different real situations. A detailed description of parameters and their usage is given so that a situation, a sequence of events, and an outlook of the future could be implemented. The simulation, not only captures the model dynamic of the spread of the virus, but also, takes care of population and mobility data. The data used was only about selected countries, but following the method adopted, the simulation can be performed for any country or region. 
The cases and results presented in this paper, provide an educated guideline to the authorities about worst- and best-case scenarios and important factor leading to corresponding outcomes. The paper also guides other researchers towards modeling such systems and why they should be careful about putting forward predictions.

\section{Acknowledgment}

The research leading to these results has no Funding.

\section{References}

[1]. H. Nishiura, H. Oshitani, T. Kobayashi, T. Saito, T. Sunagawa, T. Matsui, T. Wakita, M. COVID, M. Suzuki, Closed environments facilitate secondary transmission of coronavirus disease 2019 (covid-19), medRxiv (2020).

[2]. G. Lippi, M. Plebani, Laboratory abnormalities in patients with covid-2019 infection, Clinical Chemistry and Laboratory Medicine (CCLM) (0) (2020) 20200198. ～URL https://www.degruyter.com/view/journals/cclm/ahead-of-print/article-10.1515-cclm-2020-0198/article10.1515-cclm-2020-0198.xml.

[3]. E. Callaway, China coronavirus: labs worldwide scramble to analyse live samples, Nature (2020).

[4]. D. Wu, T. Wu, Q. Liu, Z. Yang, The sars-cov-2 outbreak: what we know, International Journal of Infectious Diseases (2020). doi:https://doi.org/10.1016/j.ijid.2020.03.004.

URL http://www.sciencedirect.com/science/article/pii/S1201971220301235

[5]. A. Wilder-Smith, C. J. Chiew, V. J. Lee, Can we contain the covid-19 outbreak with the same measures as for sars?, The Lancet Infectious Diseases (2020). doi:https://doi.org/10.1016/S1473-3099(20)30129-8. URL http://www.sciencedirect.com/science/article/pii/_S1473309920301298

[6]. C. Sohrabi, Z. Alsafi, N. O’Neill, M. Khan, A. Kerwan, A. AlJabir, C. Iosifidis, R. Agha, World health organization declares global emergency: A review of the 2019 novel coronavirus (covid-19), International Journal of Surgery 76 (2020) 71 - 76. doi:https://doi.org/10.1016/j.ijsu.2020.02.034.

URL http://www.sciencedirect.com/science/article/pii/S1743919120301977

[7]. J. Hellewell, S. Abbott, A. Gimma, N. I. Bosse, C. I. Jarvis, T. W. Russell, J. D. Munday, A. J. Kucharski, W. J. Edmunds, F. Sun, S. Flasche, B. J. Quilty, N. Davies, Y. Liu, S. Clifford, P. Klepac, 450 M. Jit, C. Diamond, H. Gibbs, K. [van Zandvoort], S. Funk, R. M. Eggo, Feasibility of controlling covid-19 outbreaks by isolation of cases and contacts, The Lancet Global Health 8 (4) (2020) e488 - e496. doi:https://doi.org/10.1016/S2214-109X(20)30074-7.

URL http://www.sciencedirect.com/science/article/pii/455S2214109X20300747

[8]. M. Chinazzi, J. T. Davis, M. Ajelli, C. Gioannini, M. Litvinova, S. Merler, A. P. y Piontti, K. Mu, L. Rossi, K. Sun, et al., The effect of travel restrictions on the spread of the 2019 novel coronavirus (covid-19) outbreak, Science (2020).

[9]. F. Brauer, C. Castillo-Chavez, C. Castillo-Chavez, Mathematical models in population biology and epidemiology, Vol. 2, Springer, 2012.

[10]. L. Perez, S. Dragicevic, An agent-based approach for modeling dynamics of contagious disease spread, International journal of health geographics 8 (1) (2009) 50.

[11]. D. Balcan, H. Hu, B. Goncalves, P. Bajardi, C. Poletto, J. J. Ramasco, D. Paolotti, N. Perra, M. Tizzoni, W. Van den Broeck, et al., Seasonal transmission potential and activity peaks of the new influenza a (h1n1): a monte carlo likelihood analysis based on human mobility, BMC medicine 7 (1) (2009) 45. 
[12]. W. Van den Broeck, C. Gioannini, B. Gon ${ }_{s}$ calves, M. Quaggiotto, V. Colizza, A. Vespignani, The gleamviz computational tool, a publicly available software to explore realistic epidemic spreading scenarios at the global scale, BMC infectious diseases 11 (1) (2011) 37.

[13]. L. Di Domenico, G. Pullano, P. Coletti, N. Hens, V. Colizza, Expected impact of school closure and telework to mitigate covid-19 epidemic in france.

[14]. "Wikipedia", "2020 coronavirus pandemic in oman".

URL https://en.wikipedia.org/wiki/2020_coronavirus_pandemic_in_Oman/

[15]. K. Zia, U. Farooq, Covid-19 outbreak in pakistan: Model-driven impact analysis and guidelines, arXiv preprint arXiv:2004.00056 (2020).

(c) 1 Author(s) and ACAA permit unrestricted use, distribution, and reproduction in any medium, provided the original work with proper citation. This work is licensed under Creative Commons Attribution International License (CC BY 4.0). 